\title{
ARTí́CULO
}

\section{Distribución, densidad, proporción sexual y fecundidad de Belzebub faxoni (Decapoda, Luciferidae) en el Sistema Arrecifal Veracruzano, SO del Golfo de México}

Distribution, density, sex ratio and fecundity of Belzebub faxoni (Decapoda, Luciferidae) in the Veracruz Reef System, SW Gulf of Mexico

\section{Sergio Cházaro-Olvera ${ }^{1 *}$, Manuel Ortiz ${ }^{1}$, Ignacio Winfield ${ }^{1}$, Jesús Alfonso Pérez-Ramos ${ }^{1}$ y Cesar Meiners-Mandujano ${ }^{2}$}

\begin{abstract}
${ }^{1}$ Laboratorio de Crustáceos, Facultad de Estudios Superiores Iztacala, Universidad Nacional Autónoma de México, Av. de los Barrios No.1, Los Reyes Iztacala, Tlalnepantla, Estado de México, México. *schazaro@gmail.com

${ }^{2}$ Instituto de Ciencias Marinas and Pesquerías, Universidad Veracruzana, Boca del Río Veracruz, México

Abstract.- The distribution, density, biomass, sex ratio and fecundity of Belzebub faxoni in the Veracruz Reef System National Park, southwest of the Gulf of Mexico, were analysed. The collection of biological samples was conducted on a motorboat with outboard motor in June, August and October 2011; January, March and May 2012; and January, March and June 2013, using a conical net of $330 \mu \mathrm{m}$. Salinity, temperature and oxygen were measured. The total number of shrimps were counted and standardized at $100 \mathrm{~m}^{-3}$. The biomass and height were measured. The number of eggs of each gravid female was counted. The distribution and density was analysed. The average temperature was $21.95 \pm 0.11$ to $29.29 \pm 0.19^{\circ} \mathrm{C}$, salinity of $33.91 \pm 0.10$ to $35.90 \pm 0.14$, oxygen $2.45 \pm 0.23$ in the northern zone and $5.63 \pm 0.61 \mathrm{mg} \mathrm{l}^{-1}$ in the south. The highest density was presented in March 2013, with 6,391 shrimp $/ 100 \mathrm{~m}^{-3}$. Generally, the highest density was presented in transects B and $D$ (centre and south). March 2013 had the highest biomass with $5,283 \mathrm{mg} 100 \mathrm{~m}^{-3}$. Females with a total length of $12.28 \pm 0.59 \mathrm{~mm}$ had a fertility rate of $7.1 \pm 3.43$ eggs. The sex ratio in general was favourable for females from 1.50:1 to $20: 1$. The main breeding season was recorded in May and June, which determined the distribution and abundance of $B$. faxoni. In addition, within the system, a changing in current direction may trigger changes in distribution. This is the first study of the population of B. faxoni in the SW Gulf of Mexico.
\end{abstract}

Key words: Gulf of Mexico, reefs, decapod, zooplankton, Belzebub faxoni

Resumen. - Se analizó la distribución, densidad, biomasa, proporción sexual y fecundidad de B. faxoni en el Parque Nacional Sistema Arrecifal Veracruzano (PNSAV), suroeste del Golfo de México. La recolección de las muestras biológicas se realizó en junio, agosto y octubre 2011; enero, marzo y mayo 2012 y enero, marzo y junio 2013. Para la recolecta se efectuaron arrastres en el nivel superficial del agua desde una lancha con motor fuera de borda, utilizando una red cónica de $330 \mu \mathrm{m}$ de abertura de malla. Se midió la salinidad, temperatura y oxígeno. Se contabilizó el número total de camarones y se estandarizó a $100 \mathrm{~m}^{-3}$. Se midió la talla y la biomasa. Se contó el número de huevos de cada hembra grávida. Se analizó la distribución y densidad. Los valores de los parámetros durante los 3 años de muestreo oscilaron entre $21,95 \pm 0,11$ a $29,29 \pm 0,19{ }^{\circ} \mathrm{C}$ de temperatura, $33,91 \pm$ 0,10 a $35,90 \pm 0,14$ de salinidad y 2,45 $\pm 0,23$ a 5,63 $\pm 0,61 \mathrm{mg} \mathrm{l}^{-1}$ de oxígeno. La mayor densidad se presentó en marzo 2013 con 6.391 camarones $100 \mathrm{~m}^{-3}$. De manera general la mayor densidad se presentó en los transectos B y D (centro y sur del PNSAV). Marzo 2013 presentó la mayor biomasa con $5.283 \mathrm{mg} 100 \mathrm{~m}^{-3}$. Las hembras con longitud total de $12,28 \pm 0,59 \mathrm{~mm}$ presentaron una fecundidad de 7,1 $\pm 3,43$ huevos. La proporción de sexos en general fue favorable a las hembras con 1.50:1 to 2 : 1 个. La principal temporada reproductiva se registró en mayo y junio lo que a su vez determinó la distribución y abundancia de B. faxoni. Además, dentro del sistema la modificación de la dirección de corrientes también modifica su distribución. Este es el primer estudio de la población de B. faxoni en el So del Golfo de México.

Palabras clave: Golfo de México, arrecifes, decápodos, zooplancton, Belzebub faxoni 


\section{INTRODUCCIÓN}

Las especies de la Familia Luciferidae De Haan, 1849 ha sido recientemente reagrupadas por Vereshchaka et al. (2016) en 2 géneros: 1) el género Lucifer J. V. Thompson, 1829 con 2 especies, Lucifer orientalis Hansen, 1919 y Lucifer typus $\mathrm{H}$. Milne Edwards, 1837; y 2) el género Belzebub Vereshchaka et al. 2016 con 5 especies: Belzebub chacei (Bowman, 1967), Belzebub faxoni (Borradaile, 1915), Belzebub hanseni (Nobili, 1905), Belzebub intermedius (Hansen, 1919), Belzebub penicillifer (Hansen, 1919).

Las especies del género Belzebub Vereshchaka, Olesen y Lunina, 2016 son holoplanctónicas y representan un componente importante en la dinámica de las redes tróficas marinas, dado que es común encontrarlas en los contenidos estomacales de peces costeros (Pascual et al. 2002) e incluso, en grandes filtradores como el tiburón ballena Rhincodon typus Smith, 1828 (Motta et al. 2010).

La especie B. faxoni tiene un tamaño aproximado desde 10,5 hasta 12,0 mm; el ojo y pedúnculo ocular son de igual o menor longitud respecto a la mitad del largo total del cuello, en los machos; el sexto segmento abdominal se estrecha hacía la punta de manera ventral (Naomi et al. 2006). El petasma, tiene una vaina subcónica, con hileras distales longitudinales de finas escamas, también se presenta un conjunto de estructuras pequeñas en forma de placa, con los bordes enteros, el proceso ventral es corto y con pocas setas; el diente exterior del exópodo del urópodo casi llega al extremo distal del exópodo (Vereshchaka et al. 2016). Belzebub faxoni se encuentra en el océano Atlántico, desde Nueva Escocia en Canadá hasta el Río Grande en el sur de Brasil, es un componente importante del plancton y es común encontrarla a profundidades de 5 a 55 $\mathrm{m}$, sin embargo, es usual que se presente en aguas costeras (D'Incao 1997). Esta especie tiene un ciclo de vida corto, altas tasas de crecimiento, reproducción continua y protege a sus huevos debajo de los somitas torácicos (Lee et al. 1992).

Se han realizado estudios sobre $B$. faxoni considerando principalmente su distribución y algunos aspectos ecológicos (Bowman \& MCcain 1967, Harper Jr 1968, Troost 1975, EPOPEM 1979, Álvarez 1985, Siokou-Frangou \& Papathanassiou 1991, Lee et al. 1992, Sankarankutty \& Barca 2000, Fernandes et al. 2002, Pascual et al. 2002, Fugimura et al. 2005, Almeda et al. 2013). Sin embargo, sobre aspectos poblacionales sólo se conocen los trabajos de López (1966) y Teodoro et al. $(2012,2014)$ realizados en costa de Brasil. Por otro lado, B. faxoni está entre las especies de decápodos con altos valores de densidad, por lo que es significativa la contribución en biomasa para el funcionamiento de ecosistemas costeros (Barba 1999), además, es un componente importante en la dieta de especies de peces de importancia comercial (Omori 1977). Por lo mencionado anteriormente, el objetivo del presente estudio fue analizar la distribución, densidad, proporción sexual y fecundidad poblacional de B. faxoni recolectada en el Parque Nacional Sistema Arrecifal Veracruzano, y contribuir con primer estudio de la población de B. faxoni en el suroeste del Golfo de México.

\section{Materiales Y MÉTODOS}

\section{Área de ESTUdio}

El Parque Nacional Sistema Arrecifal Veracruzano (PNSAV) se localiza sobre la plataforma continental en el suroeste del Golfo de México frente al Puerto de Veracruz, entre las coordenadas $19^{\circ} 00^{\prime} 00^{\prime \prime}-19^{\circ} 16^{\prime} 00^{\prime \prime N}$; 95 $45^{\circ} 00^{\prime}$ $96^{\circ} 12^{\prime} 00^{\prime} \mathrm{O}$. El parque se compone por 23 bancos arrecifales divididos por la desembocadura del río Jamapa; 12 arrecifes frente al puerto de Veracruz denominado grupo Norte y 11 arrecifes frente el Municipio de Antón Lizardo llamado grupo Sur. En total, ocupan una superficie de 52.283 hectáreas. El parque presenta bajos, islas y arrecifes de plataforma (Fig. 1) (Rodríguez-Gómez et al. 2013, Winfield et al. 2013).

El clima dentro del PNSAV es cálido-húmedo con 2 épocas bien marcadas: la época de 'nortes' que va de septiembre a abril, caracterizada por presentar temperaturas bajas y masas de aire frío provenientes de la latitud norte. La época de lluvias que se presenta de mayo a agosto, donde las temperaturas se elevan, la precipitación aumenta y los vientos son más débiles (Winfield et al. 2013). La temperatura media anual en la zona arrecifal es de $26^{\circ} \mathrm{C}$ (Chávez et al. 2007).

\section{Trabajo en campo}

La recolección de las muestras biológicas se realizó en junio, agosto y octubre 2011; enero, marzo y mayo 2012 y enero, marzo y junio 2013. Se establecieron estaciones de muestreo sobre 4 transectos perpendiculares a la costa (A, norte; B, centro-norte; C, centro-sur; D, sur) (Fig. 1). Se realizaron arrastres superficiales sobre las estaciones utilizando una red cónica de 1,5 $\mathrm{m}$ de longitud, 0,5 $\mathrm{m}$ de diámetro de la boca ( superficie $=0,196 \mathrm{~m}^{-2}$ ) y apertura de malla de $330 \mu \mathrm{m}$, a la que se le colocó un flujómetro (General Oceanics) para determinar el volumen de agua filtrado. Los arrastres se realizaron desde una embarcación con motor fuera de borda y tuvieron una duración de 5 min a una velocidad promedio de 3 nudos $\left(1.543 \mathrm{~ms}^{-1}\right)$. 


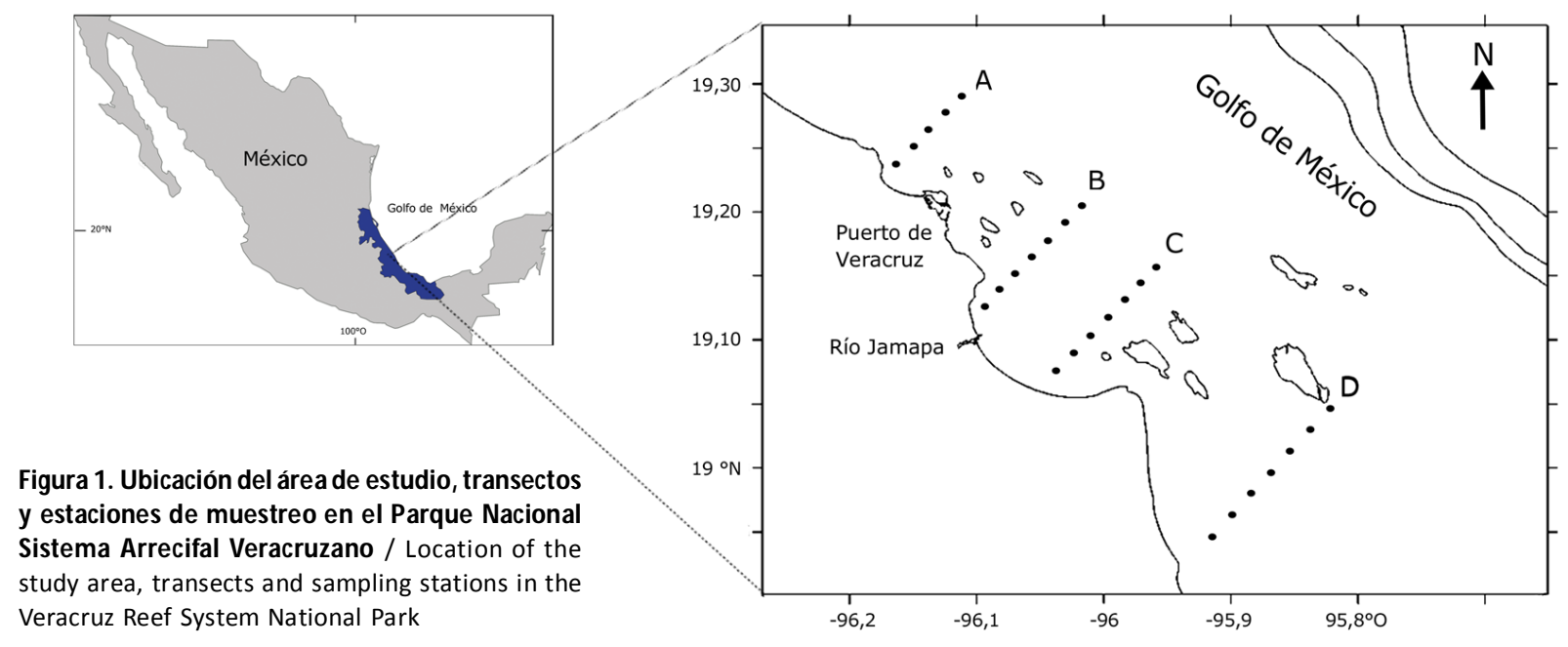

Las muestras se concentraron y fijaron en frascos de 500 $\mathrm{ml}$ con $10 \mathrm{ml}$ de formol al $4 \%$, neutralizado con borato de sodio. Después de $24 \mathrm{~h}$ el formol se cambió por alcohol al $70 \%$. Se midieron in situ la salinidad, temperatura $\left({ }^{\circ} \mathrm{C}\right)$ y oxígeno $\left(\mathrm{mg} \mathrm{l}^{-1}\right)$ con un equipo multiparamétrico (Hanna ${ }^{\circledR}$ modelo HI 9828).

\section{Trabajo de laboratorio}

El material biológico fue trasladado al Laboratorio de Crustáceos de la FES Iztacala, UNAM. Se identificaron los camarones utilizando un microscopio estereoscópico (Motic ${ }^{\circledR}$ modelo SMZ-168) y siguiendo los criterios de Williams (1984), Naomi et al. (2006) y Vereshchaka et al. (2016).

Para obtener la densidad se contabilizó el número total de camarones de B. faxoni presentes en las muestras (Mujica \& Medina 2000) y se estandarizó a número de camarones por $100 \mathrm{~m}^{-3}$, obteniendo el producto la velocidad, diámetro de la red y tiempo $\left(\mathrm{ms}^{-1} \cdot \mathrm{m}^{-2} \cdot \mathrm{s}\right)$. Para la medición de la biomasa de todos los organismos de las diferentes especies de crustáceos se empleó la técnica de Beers (1976) y para la biomasa en particular de B. faxoni se utilizó la técnica de Petruzzi (2005). Se obtuvo la talla de los camarones utilizando un calibrador con una precisión de $0,1 \mathrm{~mm}$ y el peso con una balanza analítica Ohaus ${ }^{\circledR}$ (modelo Adventurer), con una precisión de $0,0001 \mathrm{~g}$. Se contó el número total de huevos de cada hembra grávida bajo el microscopio. Para el análisis de la densidad y distribución de B. faxoni se utilizó el programa Surfer versión 10.1.561 $\left({ }^{\circledR}\right.$ Golden Software, Inc.)

\section{Análisis estadístico}

Se obtuvo el promedio de temperatura, salinidad, oxígeno, talla y densidad para cada año de muestreo \pm la desviación estándar. Los valores de temperatura, salinidad, oxígeno y densidad de B. faxoni fueron comparados entre los diferentes transectos mediante pruebas de ANDEVA de 2 factores; para la fecundidad se utilizó una prueba de 1 factor. En caso de encontrar diferencias se aplicó la prueba de Bonferroni $(P<$ $0,05)$. Para la proporción de sexos se utilizó la prueba de $\chi^{2}$ considerando la hipótesis de igualdad con las proporciones 1:1, 1.5:1 y 2:1 para cada mes de muestreo (Sokal \& Rohlf 2012). Para el cálculo de la proporción de sexos se utilizó la ecuación machos/machos+hembras (Chavance et al. 1984). Para los análisis estadísticos se utilizó el programa SigmaStat para Windows Versión 3.1 ( ${ }^{\oplus}$ Systat Software, Inc).

\section{Resultados}

\section{Temperatura, SALINIDAD y OXíGeno}

Los valores de los parámetros durante los 3 años de muestreo estuvieron entre $21,95 \pm 0,11$ y $29,29 \pm 0,19{ }^{\circ} \mathrm{C}$ de temperatura, 33,91 $\pm 0,10$ y 35,90 $\pm 0,14$ de salinidad y $2,45 \pm 0,23$ a 5,63 $\pm 0,61 \mathrm{mg} \mathrm{l}^{-1}$ de oxígeno. Se presentaron diferencias entre los meses de muestreo para la temperatura en el 2011 y 2012, para la salinidad en el 2011 y 2013 y para el oxígeno en el 2011 y 2012. Las diferencias se presentaron en los meses de primavera y verano (mayo, junio y agosto; Bonferroni=4,56; $P<0,05)$. Al comparar los valores de los parámetros entre los transectos, únicamente el oxígeno presentó diferencias significativas en los 3 años de muestreo (Bonferroni=4,35; $P<0,05$ ) (Fig. 2). 


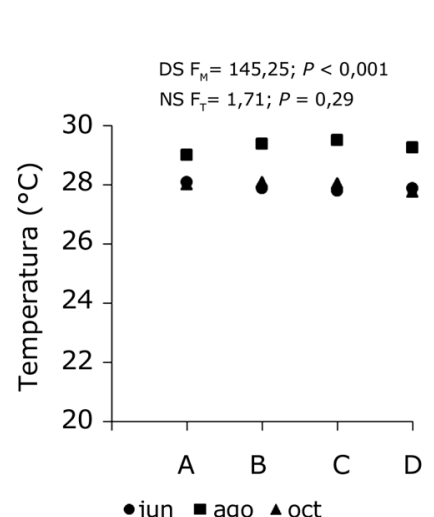

-jun - ago $\Delta$ oct

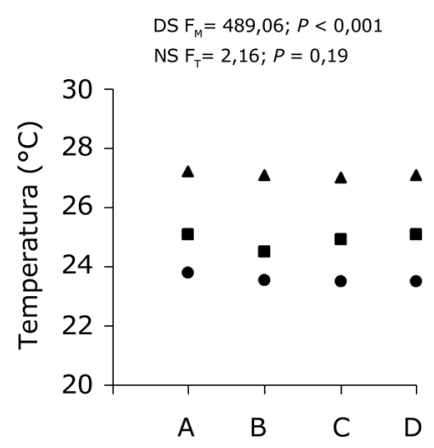

- ene - mar $\Delta$ may

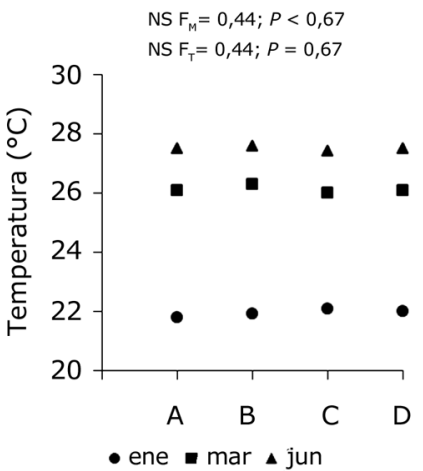

2011

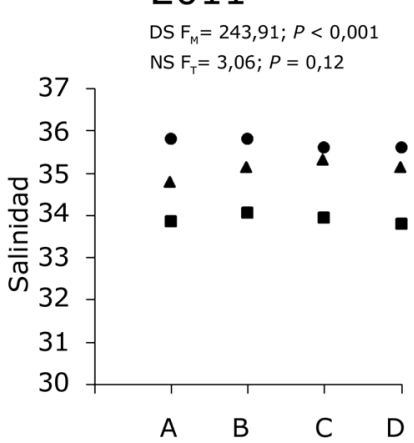

-jun - ago $\Delta$ oct

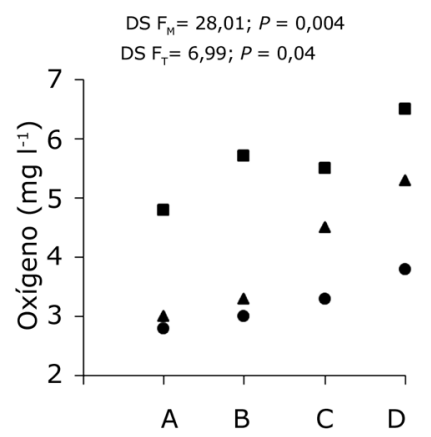

- jun - ago $\Delta$ oct

\section{2}

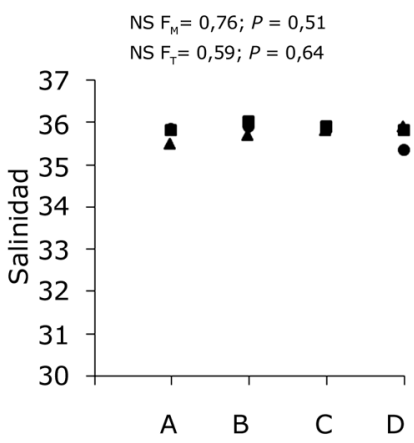

- ene a mar ^ may

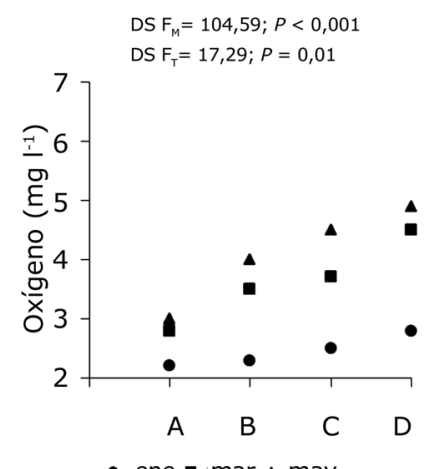

\section{3}
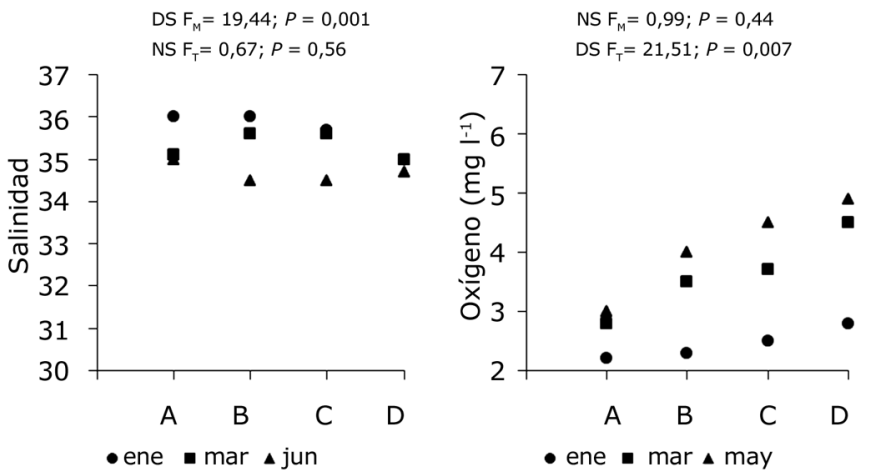

Figura 2. Valores promedio de temperatura $\left({ }^{\circ} \mathrm{C}\right)$, salinidad y oxígeno $\left(\mathrm{mg} \mathrm{l}^{-1}\right)$ en los transectos de muestreo en el Parque Nacional Sistema Arrecifal Veracruzano. FM, valor de F para meses; FT, valor de F para transectos; NS, diferencia no significativa; DS, diferencia significativa; $\mathbf{P}$, probabilidad / Average temperature $\left({ }^{\circ} \mathrm{C}\right)$, salinity and oxygen $\left(\mathrm{mg} \mathrm{I}^{-1}\right)$ sampling transects in the Veracruz Reef System National Park. FM, F value for months; FT, F value for transects; NS, not significant difference; DS, significant difference; $P$, probability 
A)

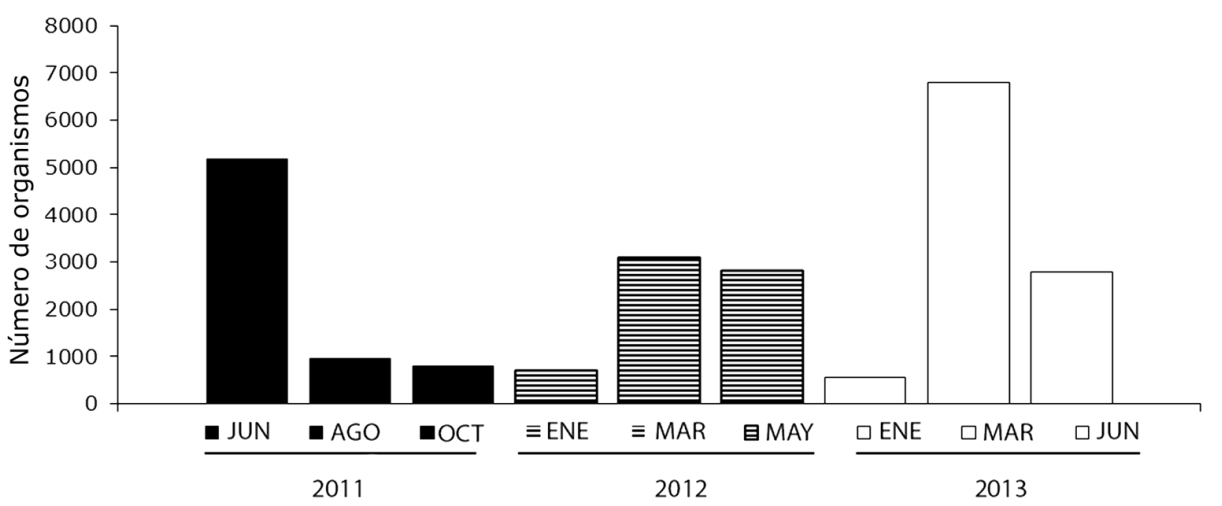

B)

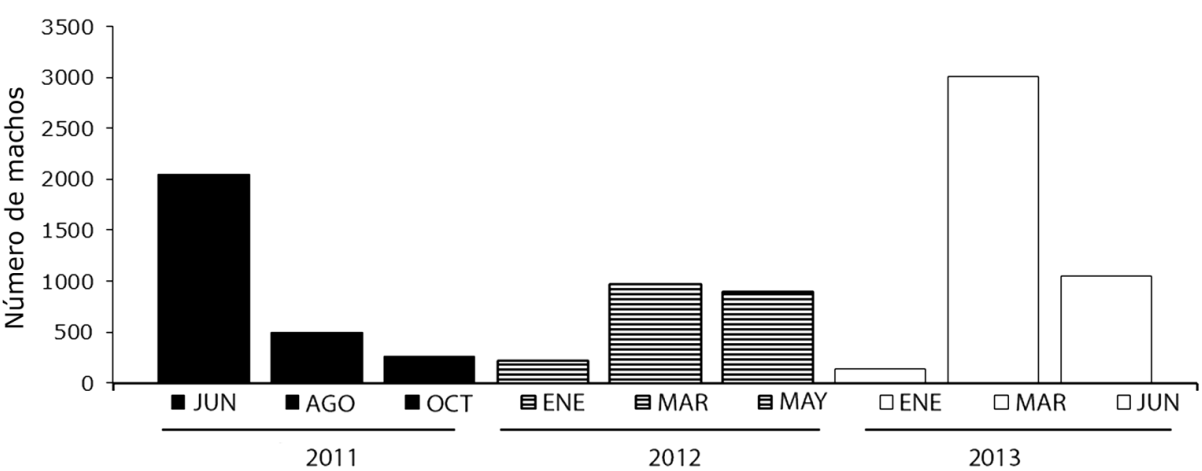

C)

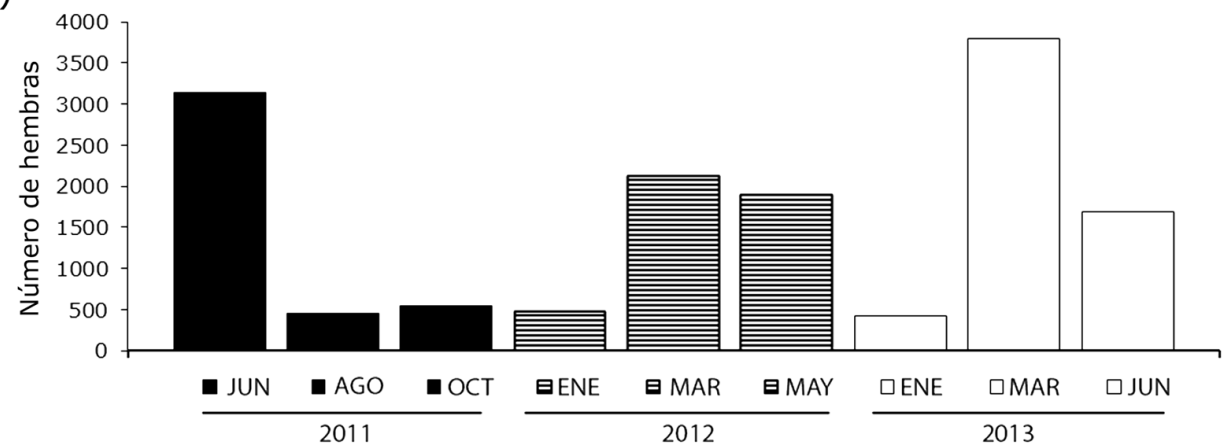

Figura 3. Número de individuos de B. faxoni por mes y año de muestreo en el Parque Nacional Sistema Arrecifal Veracruzano. A) Número total, B) Número de machos y C) Número de hembras / Number of individuals of B. faxoni by month and year of sampling in the Veracruz Reef System National Park. A) total number; B) number of males; and C) number of females

\section{Abundancia}

Se recolectaron un total de 23.666 de camarones de B. faxoni en los 3 años de muestreo. El mayor número de individuos se registró en marzo 2013 con 6.800 camarones, seguido de junio 2011 y marzo 2012 (Fig. 3A). Para ambos sexos el número mayor de individuos en presentó en marzo y junio (Figs. 3B y C).

\section{DisTRIBUCIÓN Y DENSIDAD TEMPORAL}

La mayor densidad de camarones se presentó durante marzo 2013 con 6.391 camarones $100 \mathrm{~m}^{-3}$, seguido por junio 2011 con 3.106 camarones $100 \mathrm{~m}^{-3}$. En enero 2013 se encontró la densidad más baja con 536 camarones $100 \mathrm{~m}^{-3}$. Sin embargo, no se encontraron diferencias entre los meses de los 3 años de muestreo ( $\mathrm{F}=1,96 ; P=0,27)$ (Fig. 4). 

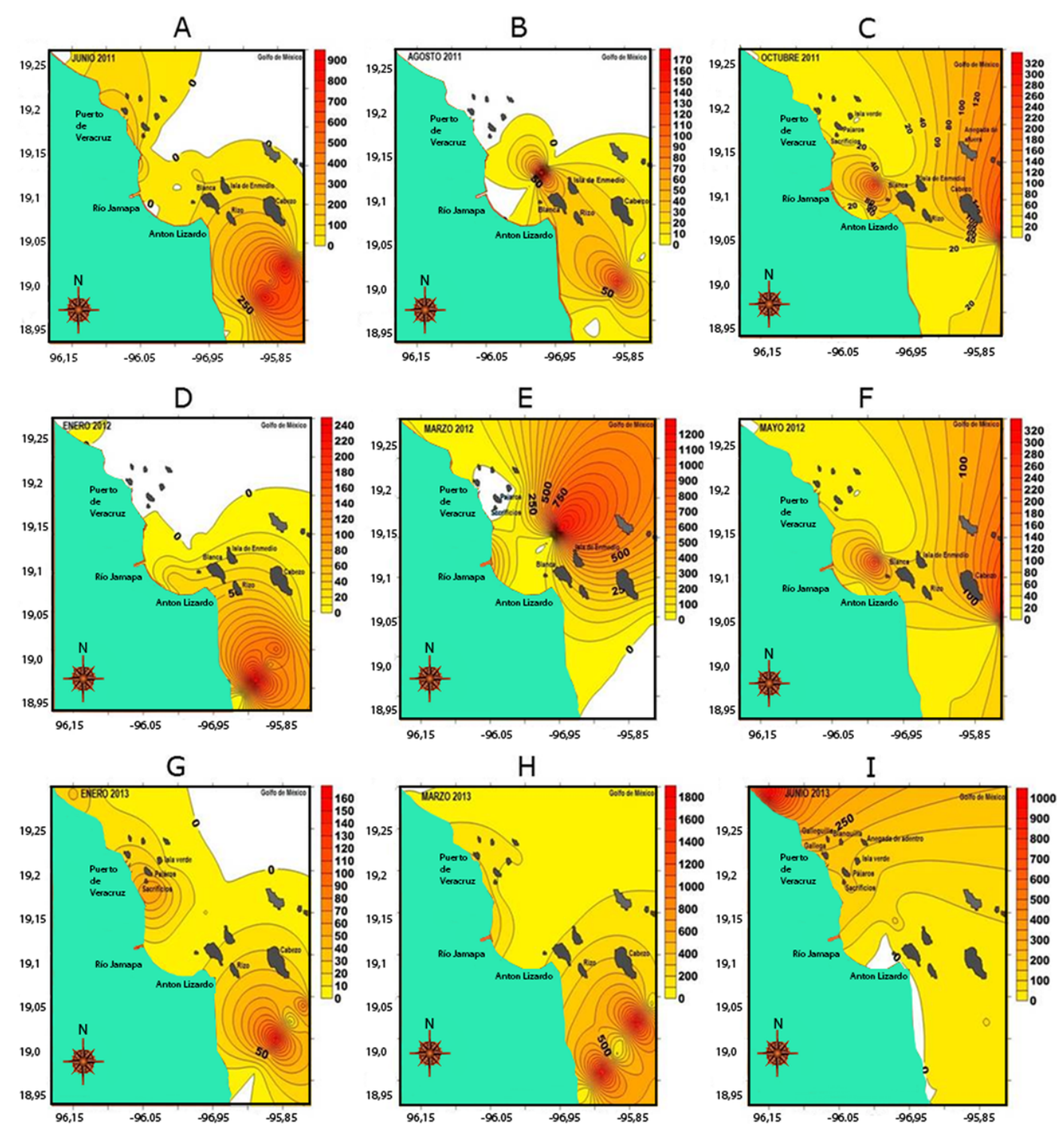

Figura 4. Distribución y densidad temporal y espacial de B. faxoni durante los meses y años de muestreo en el Parque Nacional Sistema Arrecifal Veracruzano. Rojo: mayor densidad, Amarillo: menor densidad/ Distribution, temporal and spatial density of B. faxoni in the months and years of sampling in the Veracruz Reef System National Park. Red: higher density, Yellow: minor density

\section{DisTRIBUCIÓN Y DENSIDAD ESPACIAL}

Los valores más altos de densidad en 2011 se presentaron en las zonas más alejadas de la línea de costa del transecto D (sur del PNSAV) en junio con valores entre 413 y 893 camarones $100 \mathrm{~m}^{-3}$. En 2012 se encontró la mayor densidad del transecto B (centro del PNSAV) en marzo con 1.221, 663 y 810 camarones $100 \mathrm{~m}^{-3}$. Para 2013 la mayor densidad se encontró en el transecto D (sur del PNSAV) en marzo, en las estaciones alejadas de la costa con más de 1.700 camarones $100 \mathrm{~m}^{-3}$, siendo la densidad más alta para todo el estudio (Fig. 4).

Al aplicar el análisis de varianza entre los transectos se presentaron diferencias $(\mathrm{F}=12,67 ; P<0,05)$. De manera general la distribución de $B$. faxoni se encontró con mayor densidad en los transectos B y D (Bonferroni= 5,46; $P<0,05$ ). 


\section{Biomasa}

Los valores de biomasa obtenidos para $B$. faxoni indican que en marzo 2013 se encontró el valor más alto con $5.283 \mathrm{mg}$ $100 \mathrm{~m}^{-3}$. El mes con menor biomasa fue enero 2013 con 443,09 mg $100 \mathrm{~m}^{-3}$ (Fig. 5).

Considerando a todas las especies los crustáceos del zooplancton en octubre 2011, marzo 2012 y marzo 2013 se registraron los valores más altos de biomasa con $56.218 \mathrm{mg}$ $100 \mathrm{~m}^{-3}, 133.760 \mathrm{mg} 100 \mathrm{~m}^{-3}$ y $30.496 \mathrm{mg} 100 \mathrm{~m}^{-3}$ respectivamente. Los resultados indican que durante mayo 2012 y junio 2013 B. faxoni contribuye con la mayor cantidad de biomasa de crustáceos zooplanctónicos con 23,6 y 23,15\%, respectivamente (Tabla 1 ).

\section{FeCUNDIDAD}

Durante el 2012, las hembras con una longitud total de 12,28 $\pm 0,59 \mathrm{~mm}$ presentaron los valores más altos de fecundidad con 7,1 $\pm 3,43$ huevos. Sin embargo, al comparar los valores del número promedio de huevos no se encontraron diferencias entre los 3 años de estudio ( $\mathrm{F}=2,56 ; P=0,143)$ (Fig. 6).

\section{Proporción de SEXos}

De manera general, a lo largo del estudio se encontró un mayor número de hembras que de machos. La proporción de sexos de B. faxoni dentro del PNSAV presentó una proporción sexual

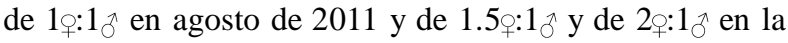
mayoría de los meses de muestreo restantes (Tabla 2).
Figura 5. Biomasa de B. faxoni durante los meses y años de muestreo en el Parque Nacional Sistema Arrecifal Veracruzano / Biomass B. faxoni in the months and years of sampling in the Veracruz Reef System National Park
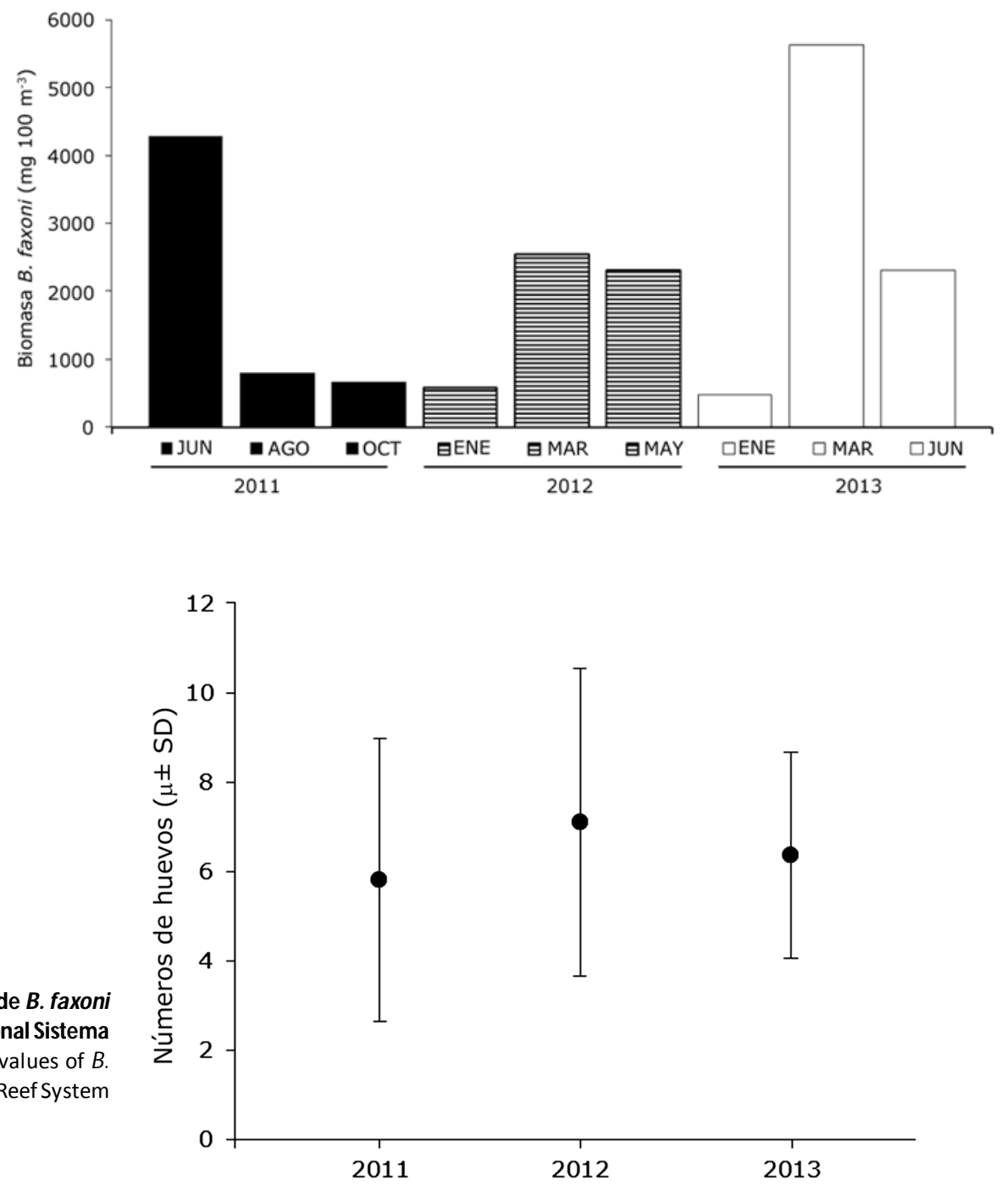
Tabla 1. Valores de biomasa mensual para B. faxoni y para todas las especies de crustáceos del zooplancton recolectadas en el Parque Nacional Sistema Arrecifal Veracruzano / Values of biomass for B. faxoni monthly and for all species of crustacean zooplankton collected in the Veracruz Reef System National Park

\begin{tabular}{ccc}
\hline & \multicolumn{2}{c}{ Valores de biomasa (mg) } \\
\cline { 2 - 3 } Mes & $\begin{array}{c}\text { Biomasa de } \\
\text { B. faxoni }\end{array}$ & $\begin{array}{c}\text { Biomasa de crustáceos } \\
\text { del zooplancton }\end{array}$ \\
\hline jun-11 & $4.281,3$ & $15.904,3$ \\
ago-11 & 785,33 & $4.563,78$ \\
oct-11 & 663,81 & $56.218,05$ \\
ene-12 & 573,7 & $4.604,4$ \\
mar-12 & $2.553,57$ & $133.760,97$ \\
may-12 & $2.306,4$ & $9.276,12$ \\
ene-13 & 466,24 & $8.924,13$ \\
mar-13 & $5.621,33$ & $30.496,09$ \\
jun-13 & $2.312,18$ & $9.225,01$ \\
Total & $19.563,86$ & $272.972,863$ \\
\hline
\end{tabular}

\section{Discusión}

Los taxones que componen el zooplancton pueden variar dependiendo la temporalidad y época del año. Sin embargo, Okolodkov (2011) encontró que 10 taxones son los más abundantes y contribuyen de manera importante en a la biomasa zooplanctónica dentro del PNSAV, entre estos taxones destacan los decápodos con un $10 \%$ promedio para cada muestreo. Al respecto, Barba (1999) encontró que $B$. faxoni representa hasta el 8,18\% del número de crustáceos capturados en la lagua Madre de Tamaulipas, en el Golfo de México, mientras que Gómez \& Hernández (2008) mencionan que la especie representó el 19,48\% de la composición del zooplancton en la costa de Isla Margarita en Venezuela. En este estudio se encontró que B. faxoni contribuyó con valores hasta del $23 \%$ de la biomasa de crustáceos del PNSAV, por lo que se puede considerar como un componente importante del zooplancton en esta región.

\section{DENSIDAD TEMPORAL}

En marzo al inicio de la primavera, comienza a incrementarse la densidad de B. faxoni en el PNSAV, lo que se relaciona con el inicio de la temporada de reproducción (Naomi et al. 2006). Las hembras ovígeras estuvieron presentes en este estudio en mayo y junio. López (1966) detectó la presencia de espermatóforos bien formados en esta temporada. Así mismo, se ha encontrado que las mayores densidades de individuos maduros e inmaduros se presentan durante la época de sequía
Tabla 2. Proporción de sexos, mensual y total de B. faxoni dentro en el Parque Nacional Sistema Arrecifal Veracruzano. $\chi^{2}$, valores de Chi cuadrada con proporciones de $1: 1,1,5: 1$ y $2,5: 1 ; P$, probabilidad; * ${ }^{*}$ in diferencia significativa / Sex ratio, total and monthly, of B. faxoni within the Veracruz Reef System National Park. $\chi^{2}$, values of Chi squared $1: 1,1.5: 1$ y $2.5: 1 ; P$, probability; * without significant difference

\begin{tabular}{crrrrrr}
\hline & \multicolumn{7}{c}{$\chi^{2}$} \\
\cline { 2 - 7 } Mes & $1: 1$ & $P$ & $1,5: 1$ & $P$ & $2,5: 1$ & $P$ \\
\hline jun-11 & 137,33 & $<0,0001$ & 0,344 & $0,558^{*}$ & 52,871 & $<0,0001$ \\
ago-11 & 0,444 & $0,505^{*}$ & 38,379 & $<0,0001$ & 105,25 & $<0,0001$ \\
oct-11 & 92,28 & $<0,0001$ & 17,417 & $<0,0001$ & 0,188 & $0,663^{*}$ \\
ene-12 & 84,133 & $<0,0001$ & 16,888 & $<0,0001$ & 0,399 & $0,527^{*}$ \\
mar-12 & 243,64 & $<0,0001$ & 29,623 & $<0,0001$ & 2,593 & $0,107^{*}$ \\
may-12 & 461,15 & $<0,0001$ & 121,95 & $<0,0001$ & 15,541 & $<0,0001$ \\
ene-13 & 134 & $<0,0001$ & 50,25 & $<0,0001$ & 16,75 & $<0,0001$ \\
mar-13 & 87,533 & $<0,0001$ & 46,139 & $<0,0001$ & 337,85 & $<0,0001$ \\
jun-13 & 142,64 & $<0,0001$ & 3,296 & $0,067^{*}$ & 28,099 & $<0,0001$ \\
Total & 1092,29 & $<0,0001$ & 21,933 & $<0,0001$ & 233,01 & $<0,0001$ \\
\hline
\end{tabular}

(Rodríguez 1973, Zoppi de Roa et al. 2008). Se ha señalado que la temporada de sequía es la más propicia para el crecimiento y desarrollo larval de B. faxoni, ya que los parámetros como la salinidad y la temperatura se encuentran más estables debido a la falta de lluvias, lo que significa una mayor tasa de sobrevivencia para las larvas y un aumento de la población adulta (Teodoro et al. 2012).

En junio se presentaron las densidades más altas de B. faxoni en el PNSAV, esto se debe a que la reproducción se inicia en la época de sequía (López 1966, Álvarez 1985, Teodoro et al. 2012). Asimismo, es en junio cuando se presentan las primeras lluvias sobre las montañas, lo que propicia una adición muy importante de nutrientes y materia orgánica por parte del río Jamapa a las aguas del PNSAV (Gutiérrez-Ruiz et al. 2011), proporcionando una mayor disponibilidad de alimento para muchas especies (Salas-Monreal et al. 2009, Okolodkov et al. 2011, Jasso 2012, Salas-Pérez et al. 2012), entre ellas $B$. faxoni, que es una especie omnívora (Lee et al. 1992) que utiliza al detritus como uno de sus ítems alimenticios y por lo que es común encontrarla en aguas ricas en nutrientes (Webber et al. 1996).

Durante el periodo de febrero a abril se han observado las densidades más altas de $B$. faxoni, destacando marzo con 1.033 individuos $100 \mathrm{~m}^{-3}$ (López 1966). Esta temporada corresponde al verano en Brasil y a la primavera en el PNSAV. Así mismo, se han encontrado densidades de 796 a 1.248 
individuos $100 \mathrm{~m}^{-3}$ de 2002 a 2005, sin mostrar diferencias estadísticamente significativas entre estos años de estudio (Gómez \& Hernández 2008). Así mismo, se han obtenido valores altos de densidad durante la primavera (2005 y 2006) con 840 y 1.112 individuos $100 \mathrm{~m}^{-3}$ (Teodoro et al. 2014), esto concuerda con lo encontrado en el PNSAV, ya que 2 de los picos de mayor densidad se presentaron en la temporada secas (primavera), sin embargo, los valores obtenidos en el presente estudio fueron mayores (2.920 a 6.391 individuos 100 $\left.\mathrm{m}^{-3}\right)$.

\section{DensiDad espacial}

De acuerdo con Castro et al. (2004), los valores de salinidad y de temperatura son determinantes en la presencia de $B$. faxoni y son similares a los encontrados en la Bahía de Sao Paulo (salinidad de alrededor de 36 y temperatura superior a $20^{\circ} \mathrm{C}$ ). Dentro del PNSAV se presentan 2 fenómenos de gran importancia que modifican la dirección de las corrientes, la temperatura y en consecuencia la distribución del plancton (Salas-Monreal et al. 2009, Salas-Pérez et al. 2008, SalasMonreal 2011, Chacon-Gómez et al. 2013); es decir, que al ser B. faxoni una especie planctónica, estos fenómenos están involucrados directamente en su distribución. El primero es un giro ciclónico que se forma justo frente a la desembocadura del Río Jamapa (transecto B), que genera menor temperatura debido al bombeo hacía la superficie de aguas más profundas, con temperaturas bajas y ricas en nutrientes, lo que se ve reflejado en áreas de alta productividad en la parte norte del giro ciclónico (Salas-Monreal et al. 2009). Como se observó en este estudio, la mayor concentración de oxígeno se registró en el transecto B, lo que explica los valores más altos en el número de individuos y la constante presencia de B. faxoni en esta área a finales de marzo y junio. El incremento en el número de individuos también se relaciona con el transporte de plancton por las corrientes (Zavala-Hidalgo et al. 2003. Salas-Pérez et al. 2008).

La baja densidad de B. faxoni en el transecto A, zona que corresponde a la parte norte del PNSAV, cerca del puerto de Veracruz, se relaciona con las bajas concentraciones de oxígeno. Robles (2013) encontró valores de 1,4 a 1,9 $\mathrm{mg} \mathrm{l}^{-1}$, mientras que en este estudio los valores fueron de 2,63 a 2,53 $\mathrm{mg} \mathrm{l}^{-1}$, siendo incluso menores a los mínimos adecuados para este tipo de ecosistemas $\left(2,7 \mathrm{mg} \mathrm{l}^{-1}\right)$ y que, de acuerdo con Stramma et al. (2008), causan estrés e incluso muerte de los macro-crustáceos marinos, lo que se refleja en una baja densidad.

La zona sur del PNSAV donde se localiza el transecto D, contiene aguas más profundas en comparación a las del grupo norte (Salas-Monreal et al. 2009). En esta zona los cambios en salinidad y temperatura son menos drásticos, por lo que es menor el estrés para los camarones (Salas-Pérez \& GranadosBarba 2008), lo anterior se refleja en un incremento en la densidad de B. faxoni en esta área. Además, junio es la temporada de transición de secas-lluvias, las corrientes van hacía el norte arrastrando una gran cantidad de materia orgánica proveniente del río Papaloapan al sur del PNSAV (Ortiz 2006, Ortiz et al. 2007), la que puede ser capturada por los arrecifes (Salas-Monreal et al. 2009) y aprovechada por B. faxoni. Otro aspecto que determina la mayor densidad en el transecto $\mathrm{D}$, es el transporte del plancton por la corriente en sentido nortesur característico de la temporada de nortes (Salas-Pérez \& Granados-Barba 2008).

\section{Biomasa}

La biomasa de zooplancton dentro del PNSAV presenta valores unimodales (Okolodkov et al. 2011); sin embargo, se presentan cambios durante y al final de la temporada de lluvias (septiembre-octubre), coincidiendo con el máximo valor de biomasa de todos los crustáceos zooplanctónicos obtenida en este este estudio durante octubre 2011.

Belzebub faxoni es una especie omnívora (Lee et al. 1996), por lo que un componente importante en su dieta es el detritus (Webber et al. 1996), así, su densidad aumenta y por ende su biomasa durante los meses en los que la presencia de materia orgánica dentro del PNSAV es alta. La concentración de nutrientes aumenta por la aportación del cauce de los ríos ' $\mathrm{La}$ Antigua, Jamapa y Papaloapan' durante la temporada de lluvias (Gutiérrez-Ruiz et al. 2011) con valores altos de biomasa de B. faxoni (junio 2011 y mayo 2012). Como se mencionó anteriormente, el aumento en la densidad puede relacionarse con el giro ciclónico que ahí se presenta y genera afloramientos de nutrientes procedentes del fondo marino (Ortiz 2006, Ortiz et al. 2007, Salas-Monreal 2009). Zoppi de Roa et al. (2008) mencionan que la formación de núcleos de concentración de individuos de B. faxoni dependen del proceso de mezcla de las aguas fluviales y oceánicas.

\section{Fecundidad}

El número de huevos reportado para esta especie es muy variable. Woodmansee (1966) en un estudio realizado en el norte del Golfo de México menciona que el número de huevos por hembra puede variar de 30 hasta 139 con un valor promedio de 27,5 huevos. Otros estudios han encontrado de 1 a 19 huevos (López 1966), de 8 a 30 huevos por hembra (Lee et al. 1992), de 3 a 29 huevos (Fugimura et al. 2005), y de 1 a 13 huevos por hembra (Teodoro et al. 2012). En el presente 
estudio el número de huevos encontrado fue bajo ( 5 a 7), la diferencia se debe principalmente a la pérdida de los huevos durante la colecta y fijación, ya que estos se deprenden fácilmente durante la manipulación (López 1966, Teodoro et al. 2012).

\section{Proporción de SEXos}

Una proporción sexual favorable a las hembras como se presentó en la mayor parte de los meses de muestreo en este estudio, se debe a la migración que realizan durante la noche a una profundidad mayor de $15 \mathrm{~m}$, esta es una estrategia de sobrevivencia para evitar a los depredadores, buscar mayor cantidad de alimento y brindar una mayor protección a los huevos. Smith \& Smith (2006) mencionan que el sesgo hacia las hembras suele ser normal para algunos microcrustáceos holopláncticos, lo que se debe en general a que la esperanza de vida de los machos es más corta que la de las hembras.

Las hembras también suelen agruparse en áreas con mayor concentración de recursos alimenticios (Lee et al. 1992) como se observó en este estudio al interior y a la periferia del PNSAV. La proporción de sexos obtenida concuerda con lo reportado por López (1966), Cardoso (2002) y Teodoro et al. (2014) quienes encontraron una proporción de sexos favorable a las hembras en la costa de Sao Paulo y Cananéia, Brazil, respectivamente. En otros sergéstidos como Acetes americanus Ortmann, 1893 también se ha encontrado una proporción de sexos favorable a las hembras (Simões et al. 2013).

\section{Agradecimientos}

Los autores agradecemos el apoyo por los fondos otorgados para esta investigación al programa PAPIIT IN220715 de la Universidad Nacional Autónoma de México; a las autoridades de la SEMARNAT, CONANP y CONAPESCA-DGOPA (SAGARPA) por los permisos otorgados para la realización del presente estudio y a los estudiantes del laboratorio de Crustáceos de la FES Iztacala que participaron en la revisión y separación de muestras.

\section{LITERATURA CITADA}

Almeda R, Z Wambaugh, Z Wang, C Hyatt, Z Liu \& EJ Buskey. 2013. Interactions between zooplankton and crude oil: toxic effects and bioaccumulation of polycyclic aromatic hydrocarbons. PloS One 8(6): e67212. <doi: 10.1371/ journal.pone.0067212>

Álvarez MPJ. 1985. Migração vertical de Lucifer faxoni Borradaile, 1915 (Crustacea-Decapoda) nas águas ao largo de Santos, Brasil. Boletim do Instituto Oceanográfico 9: 177193.
Beers JR. 1976. Volumetric methods. In: Steedman HF (ed). Zooplankton fixation and preservation. Monographs on oceanographic methodology 4: 56-60. UNESCO, Paris.

Bowman TE \& JC McCain. 1967. Distribution of the planktonic shrimp Lucifer in the western North Atlantic. Bulletin of Marine Science 17: 660-671.

Cardoso IA. 2002. Taxonomia e distribuição do gênero Lucifer (Decapoda, Sergestoidea) na Costa Central Brasileira. Dissertação Mestrado em Ciências Biológicas-Zoologia, Universidade Federal do Rio de Janeiro, Rio de Janeiro, 53 pp.

Castro RH, RC Costa, A Fransozo \& FLM Mantelatto. 2004. Population structure of the seabob shrimp Xiphopeneus kroyeri (Heller, 1962) (Crustacea: Panaenoidea) in the litoral of São Paulo, Brazil. Scientia Marina 69: 105-112.

Chacon-Gomez IC, D Salas-Monreal \& ML RiveronEnzastiga. 2013. Current pattern and coral dispersion in a tropical coral reef system. Continental Self Research 68: 23-32.

Chavance P, D Flores-Hernández, A Yáñez-Arancibia \& F Amezcua-Linares. 1984. Ecología, biología y dinámica de las poblaciones de Bairdiella chrysoura en la Laguna de Términos, Sur del Golfo de México. (Pisces: Scianidae). Anales del Instituto de Ciencias del Mar y Limnología, Universidad Nacional Autónoma de México 11(1): 123-162.

Chavez E, JW Tunnell \& K Withers. 2007. Reef zonation and ecology. In: Tunnel JW, EA Chávez \& K Withers (eds). Coral reefs of Southern Gulf of México, Cap. 4: 41-67. Texas University Press, Houston.

EPOPEM. 1979. Systeme planctonique et pollut on urbalne. Un aspect des populations zooplanctoniques. Acta Oceanologica 2(4): 379-388.

Fernandes LDA, SLC Bonecker \& JL Valentin. 2002. Dynamic of decapod crustacean larvae on the Entrance of Guanabara Bay. Brazilian Archives of Biology and Technology 45(4): 491-498.

Fugimura SMM, LMY Oshiro \& R Silva. 2005. Distribuição e abundância das Famílias Luciferidae e Sergestidae (Crustacea, Decapoda, Natantia) na Baía de Sepetiba, Rio de Janeiro, Brasil. Revista da Universidade Rural, Série Ciências da Vida 25(2): 52-59.

Gómez A \& I Hernández. 2008. Abundancia interanual del zooplancton nocturno en la costa este de isla Margarita, Venezuela. Boletín del Instituto Oceanográfico de Venezuela 47 (2): 91-102.

Harper DE Jr. 1968. Distribution of Lucifer faxoni (Crustacea: Decapoda: Sergestidae) in neritic waters off the Texas Coast, with a note on the occurrence of Lucifer typus. Contributions in Marine Science 13: 1-16.

Jasso MJ. 2012. Variación de los parámetros oceanográficos alrededor del Arrecife Verde en el Parque Nacional Sistema Arrecifal Veracruzano (Golfo de México Occidental). Tesis 
de Maestría en Ecología y Pesquerías, Universidad Veracruzana, Boca del Río, Veracruz, 71 pp.

Lee WY, M Omori \& RW Peck. 1992. Growth, reproduction and feeding behavior of the planktonic shrimp, Luciferfaxoni Borradaile 1915, off the Texas Coast. Journal of Plankton Research 14: 61-69.

López MT. 1966. Biología de Lucifer faxoni Borradaile, 1915 en Cananéia, Brasil (Crustacea, Decapoda, Luciferidae). Boletim do Instituto Oceanográfico 15: 47-54.

Motta PJ, M Maslank, RE Hueter, RL Davis, R de la Parra, SL Mulvany, ML Habegger, JA Strother, KR Mara, JM Gardiner, JP Tyminski \& LD Zeigler. 2010. Feeding anatomy, filter-feeding rate, and diet of whale sharks Rhincodon typus during surface ram filter feeding off the Yucatan Peninsula, Mexico. Zoology 113: 199-212.

Mujica A \& M Medina. 2000. Distribución y abundancia de larvas de crustáceos decápodos en el zooplancton de canales australes. Proyecto CIMAR-Fiordo 2. Ciencia y Tecnología Marina 23: 49-68.

Naomi TS, A Geetha, RM George \& S Jasmine. 2006. Monograph on the planktonic shrimps of the genus Lucifer (Family Luciferidae) from the Indian EEZ. Central Marine Fisheries Research Institute Bulletin 49: 1-54.

Omori M. 1977. Distribution of warm water epiplanktonic shrimps of the genera Lucifer and Acetes (Macrura, Penaeidea, Sergestidae). Proceedings of the Symposium on Warm Water Zooplankton. Special Publication of UNESCO/ NIO, National Institute of Oceanografy, Goa, India, pp. 112.

Okolodkov YB, JA Aké-Castillo, MG Gutiérrez-Quevedo, H Pérez-España \& D Salas-Monreal. 2011. Annual cycle of the plankton biomass in the National Park Sistema Arrecifal Veracruzano, southwestern Gulf of Mexico. In: Kattel G (ed). Zooplankton and phytoplankton: Types, characteristics and ecology, pp. 63-88. Nova Science Publishers, New York.

Ortiz LLD. 2006. Análisis crítico de las zonas de regulación y planeación del Parque Nacional Sistema Arrecifal Veracruzano. Tesis de Doctor en Ciencias y Oceanografía Costera, Facultad de Ciencias Marinas, Universidad Autónoma de Baja California, México, 200 pp.

Ortiz LLD, AG Barba \& IE Carbajal. 2007. El contexto regional del Parque Nacional Sistema Arrecifal Veracruzano y las implicaciones para su manejo. Investigaciones Científicas del Sistema Arrecifal Veracruzano, Universidad Autónoma de Campeche, México, pp. 275-286.

Pascual CR, Y Wakabar \& MN Flynn. 2002. Composition, distribution and temporal variation of a suprabenthic community of Ribeira Bay, Angra dos Reis, Southeastern Brazil. Oceánides 17: 41-56.

Petruzzi HJ, NP Stritzler, CM Ferri, JH Pagella \& CM Rabotnikof. 2005. Determinación de materia seca por métodos indirectos: utilización de horno a microondas. Boletín de Divulgación Técnica 88: 1-11.
Robles CM. 2013. Relación de los parámetros oceanográficos con la distribución de clorofila-a en la zona norte del Parque Nacional Sistema Arrecifal Veracruzano, Golfo de México Occidental. Tesis de Maestría en Ecología y Pesquerías, Instituto de Ciencias Marinas y Pesquerías, Universidad Veracruzana, México, 59 pp.

Rodríguez G. 1973. El Sistema de Maracaibo, 395 pp. Instituto Venezolano de Investigaciones Científicas, Caracas.

Rodríguez-Gómez CF, JA Castillo \& GC Bautista. 2013. Productividad primaria bruta y respiración planctónica en el Parque Nacional Sistema Arrecifal Veracruzano. Revista Hidrobiológica 23(2): 143-153.

Salas-Monreal D, DA Salas-de-León, MA Monreal-Gómez \& ML Riverón-Enzástigo. 2009. Current rectification in a tropical coral reef system. Coral Reefs 28:871.<doi10.1007/ s00338-009-0521-9>

Salas-Pérez JJ \& A Granados-Barba. 2008. Oceanographic characterization of the Veracruz reef system. Atmosfera 21 : 281-301.

Salas-Pérez JJ, D Salas-Monreal, VEA Fuentes, DA Salasde-León \& MLR Enzastiga. 2008. Tidal characteristics in a coral reef system from the western Gulf of Mexico. Ciencias Marinas 34: 467-478.

Salas-Pérez JJ, D Salas-Monreal, MA Monreal-Gómez, ML Riveron-Enzástiga \& C Llasat. 2012. Seasonal absolute acoustic intensity, atmospheric forcing and currents in a tropical coral reef system. Estuarine Coastal and Shelf Science 100: 102-112.

Sankarankutty C \& FEN Barca. 2000. Some observations on Lucifer faxoni Borradaile (Crustacea: Decapoda; Sergestidae) collected from a tidal estuary. Journal of the Marine Biological Association of India 41(1): 103-106.

Simões SM, F D'Incao, A Fransozo, AL Castilho \& RC Costa. 2013. Sex ratio, growth and recruitment of the pelagic shrimp Acetes americanus on the southeastern coast of Brazil. Journal of Crustacean Biology 33: 1-9.

Siokou-Frangou I \& E Papathanassiou. 1991. Differentiation of zooplankton populations in a polluted area. Marine Ecology Progress Series 76: 41-51.

Sokal RR \& FJ Rohlf. 2012. Biometry: the principles and practice of statistics in biological research, 937 pp. W. H. Freeman, New York.

Stramma L, GC Johnson, J Sprintall \& V Mohrholz. 2008. Expanding oxygen-minimum zones in the Tropical Oceans. Science 320: 655-657.

Teodoro SSA, ML Negreiros-Fransozo, SM Simões, M Lopes \& RC da Costa. 2012. Population ecology of the planktonic shrimp Lucifer faxoni Borradaile, 1915 (Crustacea, Sergestoidea, Luciferidae) of the southeastern coast of Brazil. Brazilian Journal of Oceanography 60(2): 245-253. 
Teodoro SSA, JAF Pantaleão, ML Negreiros-Fransozo \& RC da Costa. 2014. Ecological aspects and sexual maturity of a southwestern Atlantic population of the planktonic shrimp Lucifer faxoni (Decapoda: Sergestoidea). Journal of Crustacean Biology 34(4): 422-430.

Troost DG. 1975. The sergestid shrimp Lucifer in the 1970 Cicar plankton samples taken by H. M. S. 'Luymes', with notes on the Amazon River as a nutrient source. Bulletin Zoologisch Museum Universiteit van Amsterdam 24(4): 201211.

Vereshchaka AL, J Olesen \& AA Lunina. 2016. A phylogeny-based revision of the family Luciferidae (Crustacea: Decapoda). Zoological Journal of the Linnean Society 178: 15-32.

Webber MK, JC Roff, LA Chisholm \& C Clarke. 1996. Zooplankton distributions and community structure in an area of the south coast shelf of Jamaica. Bulletin of Marine Science 59: 259-270.
Williams AB. 1984. Shrimps, lobsters, and crabs of the Atlantic coast of the Eastern United States, Maine to Florida, 550 pp. Smithsonian Institution Press, Washington.

Winfield I, MA Ávila, M Ortiz \& MAL Aburto. 2013. Tanaidáceos (Peracarida: Tanaidaceae) del Parque Nacional Sistema Arrecifal Veracruzano: listado faunístico y registros nuevos. Revista Mexicana de Biodiversidad 84(1): 471-479.

Woodmansee RA. 1966. Daily vertical migration of Lucifer planktonic numbers in relation to solar and tidal cycles. Ecology 47(5): 847-850.

Zavala-Hidalgo J, SL Morey \& JJ O’Brien. 2003. Seasonal circulation on the western shelf of the Gulf of Mexico using a high-resolution numerical model. Journal of Geophysical Research 108(19): 1-19.

Zoppi de Roa E, YJ Díaz, B Marin \& B Márquez. 2008. Variación espacial y temporal del zooplancton en la plataforma deltana venezolana. Revista Cubana de Investigaciones Pesqueras 25(1): 64-74.

Recibido el 13 de octubre de 2016 y aceptado el 21 de julio de 2017

Editor: Dr. Claudia Bustos / col. Dr. Mariano Diez 